\title{
Receptor Recognition by Porcine Circovirus 2
}

\author{
Shanshan Feng ${ }^{1}$, Angelica Schreyer ${ }^{1}$ and Reza Khayat ${ }^{1}$ \\ 1. Department of Chemistry, The City College of New York, 160 Convent Avenue, New York, New \\ York 10031, United States.
}

The genetics, physiology, and immunology shared between humans and swine allow for swine pathogens to be an impeding threat to human health. Porcine Circovirus 2 (PCV2) is a globally present immunosuppressive virus demonstrated to infect and replicate within human cells in culture. The capacity of PCV2 to infect nearly every tissue in its host, and jump the species barrier to raise tremendous concern that PCV2 may become zoonotic. Moreover, PCV2's ability to infect nearly every tissue in its host makes it a deterrent to xenotransplantation. Xenotransplantation from swine to humans is becoming a practiced solution for the shortage of organs from human donors. PCV2 is also the smallest known autonomous pathogen and thus defines the limit of genetic/biochemical information needed by a pathogen. Thus there are a number of medical and academic justifications to study the interaction between PCV2 and its host.

The promiscuous nature of PCV2 is unique and provides the opportunity to understand factors defining virus tropism. We are currently studying the mechanism by which PCV2 recognizes and interacts with its receptor heparan sulfate [1]. Heparan sulfate is a highly negative charged proteoglycan present on nearly all cells, and could explain how PCV2 infects so many different cell types. Our crystal structure of PCV2 capsid provided us a scaffold to begin studying the mechanism of cellular recognition and entry at atomic resolution [2]. Using a docking server and surface analysis of the PCV2 capsid identified a number of sites where heparan sulfate may bind to the capsid. Using heparin conjugated chromatography resin we have been able to demonstrate the interaction between PCV2 capsids and heparin -a chemical analogue of heparan sulfate.

The interaction appears to be: 1) slow and nearly irreversible with a 24hour period, and 2) $\mathrm{pH}$ and salt sensitive. Competition experiments with other proteoglycans and substituents reveals that PCV2 binds to negatively charged proteoglycans but does not distinguish the backbone carbohydrate chain. Using site directed mutagenesis of the capsid we have identified distal sites that are capable of binding to heparin. We are conducting atomic resolution cryo-electron microscopy (Cryo-EM) experiments to visualize the interaction between heparin and PCV2, and to identify if any structural changes occur to the PCV2 capsid upon binding to its receptor. Such conformational changes may be necessary for the successful infection of the cell. Samples will be prepared by allowing excess heparin to interact with PCV2 capsids for more than 24 hours on ice. The sample will then be buffer exchanged to remove the excess heparin and vitrified grids for cryo-EM will be prepared using an FEI Vitrobot Mark IV. Data will be collected on an FEI Titan Krios equipped with a Falcon 2 direct electron detector. Image processing will be carried out using Sparx for generating reference free class averages and Relion for generating asymmetric 3D class averages. It may be possible that heparin binds asymmetrically to the PCV2 capsid, or causes the PCV2 capsid to become asymmetric. 


\section{References:}

[1] Misinzo G, Delutte PL, Meerts P, Lefebvre DJ, Nauwynck HJ. J. Virol 2006, Apr; 80(7):3487-94. [2] Khayat R, Brunn N, Speir JA, Hardham JM, Ankenbauer RG, Schneemann A, Johnson JE. J. Virol 2011, Aug; 85(15):7856-62.

[2] The authors acknowledge funding from the National Institutes of Allergy and Infectious Diseases (NIH) Grant number 5SC1AI11483-02.
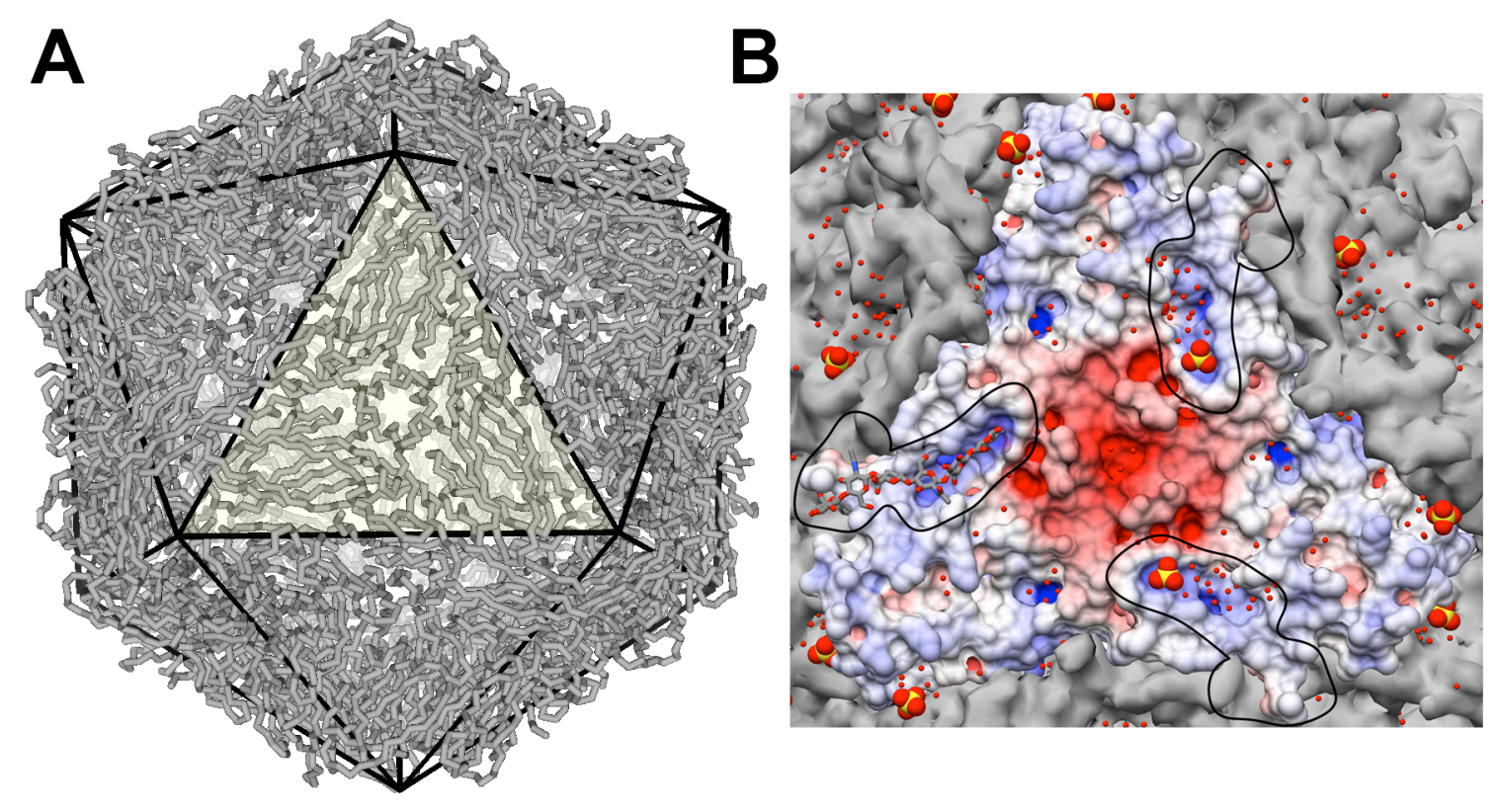

Figure 1. PCV2 receptor recognition. A) A C-alpha trace of the PCV2 crystal structure. Overlaid is a trace of the icosahedral facets. Highlighted facet is enlarged in B. B) Electrostatic potential map of an icosahedral facet showing sulfates and waters modeled into the crystal structure electron density map. A hexasaccharide heparin could be modeled into a shallow depression on the surface of PCV2 (outlined). 